\title{
NOUVELLE
}

\section{Activation de caspases et formation de dégénérescences neurofibrillaires dans un modèle murin de la maladie d'Alzheimer}

Alix de Calignon, Tara L. Spires-Jones, Bradley T. Hyman
Mass. General Institute for Neurodegenerative Disease, Building 114, $16^{\text {th }}$ Street, Charlestown, MA 02129, États-Unis. adecalig@gmail.com

\section{La maladie d'Alzheimer}

Depuis la description du premier patient par Aloïs Alzheimer en 1907, la maladie d'Alzheimer s'est révélé être la plus commune des démences séniles. Cette maladie neurodégénérative évolue lentement vers un déclin de la mémoire et des fonctions cognitives et retentit sur le comportement social des patients. Le diagnostic formel n'est possible qu'après la mort avec l'identification, dans le cerveau, de plaques extracellulaires constituées d'agrégats de peptides amyloïdes bêta $(A \beta)$, de dégénérescences neurofibrillaires (DNF) intraneuronales constituées d'agrégats de protéines tau, ainsi que de perte neuronale [1].

Bien que la recherche sur des mécanismes sous-jacents ait progressé au cours des deux dernières décennies, à commencer par la découverte de peptides $A \beta$ dans les plaques et de protéines tau hyperphosphorylées dans les DNF [14], la communauté scientifique peine à comprendre cette maladie dévastatrice. Jusqu'à présent, aucun traitement curatif n'est disponible et les symptômes ne peuvent être qu'atténués. Combattre efficacement la maladie d'Alzheimer impose de comprendre la responsabilité de ces deux types d'agrégats de protéines dans la mort neuronale et le déclin cognitif.

\section{La pathologie fibrillaire}

Bien que les plaques amyloïdes aient d'abord été mises en cause pour leur rôle fondamental dans le développement de la maladie d'Alzheimer [2], de plus en plus d'études montrent l'implication cruciale des DNF. L'importance de la protéine tau dans le neurone est majeure puisque son rôle est d'organiser et de stabiliser les microtubules de l'axone. Ce support structural est déterminant non seulement pour la morphologie neuronale mais aussi pour le trafic cellulaire et la fonction synaptique.

Les DNF sont aussi retrouvées dans de nombreuses maladies neurodégénératives appelées tauopathies - dont fait partie la démence fronto-temporale avec parkinsonisme liée au chromosome 17 (FTDP-17) - et associées à des mutations du gène tau [3]. Ces mutations, responsables de l'agrégation pathologique de la protéine tau, ont permis de recréer cet aspect de la maladie d'Alzheimer dans des souris transgéniques. Des études stéréologiques post-mortem ont montré que les agrégats de protéines tau sont retrouvés dans les régions du cerveau affectées par une perte neuronale massive [4], et que leur présence coïncide avec celle d'anomalies cellulaires [5] et d'une activité des enzymes caspases $[6,7]$. Ces marqueurs sont classique- ment associés à la mort cellulaire, ce qui suggère que l'agrégation de tau provoque la mort des neurones.

\section{Identification de la tauopathie}

fibrillaire par microscopie

\section{biphotonique in vivo}

Pour vérifier cette hypothèse, nous avons mis au point une technique permettant de visualiser la tauopathie fibrillaire dans des souris transgéniques $\operatorname{Tg} 4510$ [8] porteuses du gène tau humain muté (P301L). Grâce à des marqueurs fluorescents appliqués directement sur la surface du cerveau, nous avons pu suivre l'évolution de la pathologie par microscopie biphotonique à travers une fenêtre crânienne [9], au cours de séances d'imagerie répétées quotidiennement sur des souris sous anesthésie (Figure IA). Une solution de thioflavine $S$ permet de visualiser les DNF [10], un indicateur marque les neurones dans lesquels les caspases sont activées [11], et un colorant fluorescent liant I'ADN marque les neurones présents dans la région observée [12]. Avec cette méthode, notre but était d'observer la relation temporelle entre la présence d'une DNF dans un neurone, l'activation de caspases et la mort du neurone, afin de déterminer s'il existe un lien de cause à effet entre le marqueur de la maladie et la dégénérescence. 


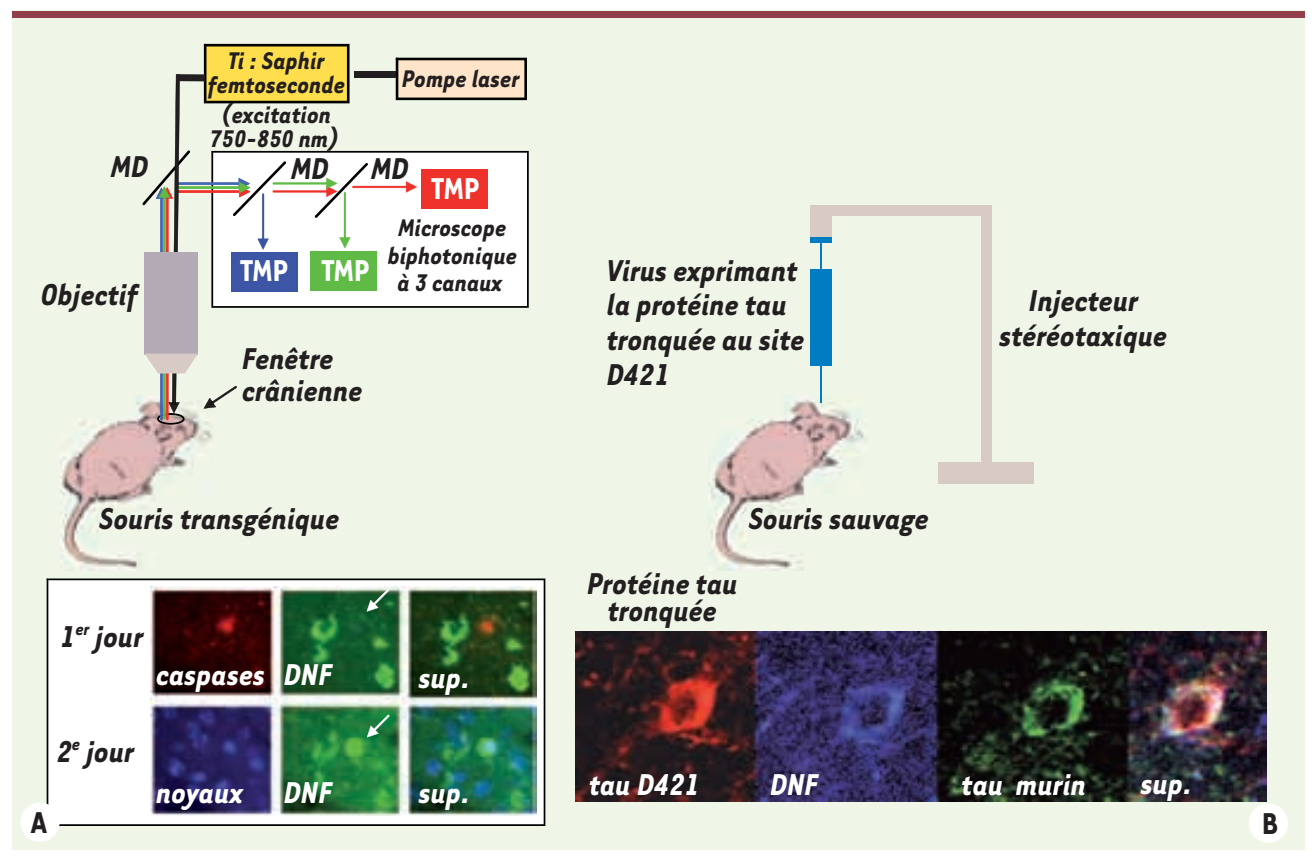

Figure 1. Relation temporelle entre activation des caspases et formation de fibrilles. A. La thioflavine $S$ (verte), le marqueur d'ADN (bleu) et l'indicateur de caspases actives (rouge) ont été appliqués directement sur la surface du cerveau de souris après craniotomie, et les neurones marqués ont été photographiés au premier jour avec un microscope biphotonique. Les mêmes neurones ont été à nouveau photographiés le jour suivant. La formation de DNF (vert, flèche) a été observée en 24 heures dans des neurones dont les caspases (marquées en rouge) étaient actives la veille. Ces neurones possédaient un noyau (bleu) confirmant leur survie. B. Un virus exprimant la protéine tau tronquée (en rouge), injecté dans le cerveau de souris sauvages, induit la formation d'agrégats de protéines tau (en bleu), ainsi que le recrutement de protéines tau endogènes (en vert).

Nos résultats, publiés dans la revue Nature en avril 2010 [13], ont montré que la présence de caspases actives est un événement rare et associé à la présence de DNF dans le neurone. En effet, la grande majorité des caspases actives a été observée dans des neurones porteurs de DNF, mais seulement dans une petite fraction de cette population. Les neurones porteurs de DNF, y compris ceux dont les caspases étaient actives, étaient stables. En effet, tous ont pu être suivis par imagerie pendant plusieurs jours. En revanche, de rares neurones indiquaient uniquement la présence de caspases actives, sans la présence de DNF. Contrairement à toute attente, l'observation longitudinale de ces rares neurones a révélé l'apparition, le jour suivant, de lésions neurofibrillaires dans ces mêmes neurones, toujours vivants (Figure IA). La formation de nouvelles lésions neurofibrillaires a été observée quasi-exclusivement dans les neurones dont les caspases étaient actives. Ces résultats suggèrent que la formation de nouvelles DNF est rapide, qu'elle est précédée par l'activation de caspases, et qu'elle ne provoque pas la mort du neurone à court terme.

\section{Les causes et conséquences}

\section{de l'activation de caspases}

Pour déterminer si la présence de la protéine tau soluble était la forme responsable de cette induction de caspases, un traitement à la doxycycline a permis, chez ces souris porteuses d'un transgène inductible, de réprimer le promoteur tétracycline et l'expression du transgène. L'analyse a montré qu'après ce traitement qui réduit la quantité de protéines tau solubles sans affecter les agrégats, le nombre de neurones présentant des caspases actives avait beaucoup diminué. Ce résultat suggère que les protéines tau sous forme soluble plutôt que fibrillaire pourraient être responsables de l'activation de caspases. Les caspases ont la propriété de cliver de nombreuses protéines, dont tau (au site D421). Pour déterminer si l'induction des DNF par les caspases passe par le clivage de tau, nous avons recherché, par marquage immunohistochimique avec un anticorps reconnaissant spéci- fiquement ce site de clivage, la présence du fragment de tau dans le cerveau des souris. La protéine tau clivée a ainsi été localisée dans les neurones dont les caspases étaient actives. Afin de vérifier que le clivage de tau par les caspases est suffisant pour induire l'agrégation de tau, nous avons injecté dans le cerveau de souris sauvages un virus exprimant la protéine tau tronquée au niveau du site de clivage (Figure 1B). La présence de ce fragment de protéine tau a provoqué la formation de dépôts visibles de tau et un changement dans la conformation de la protéine, signe précoce de la formation de DNF. En outre, la protéine tau endogène murine a été détectée dans ces mêmes dépôts fibrillaires. Ces résultats suggèrent que le clivage de la protéine tau par les caspases accélère son agrégation dans une conformation qui peut, à son tour, recruter des molécules tau supplémentaires.

\section{Conclusion et hypothèse}

Contrairement aux méthodes traditionnelles de stéréologie qui tirent leurs conclusions de la comparaison de groupes 


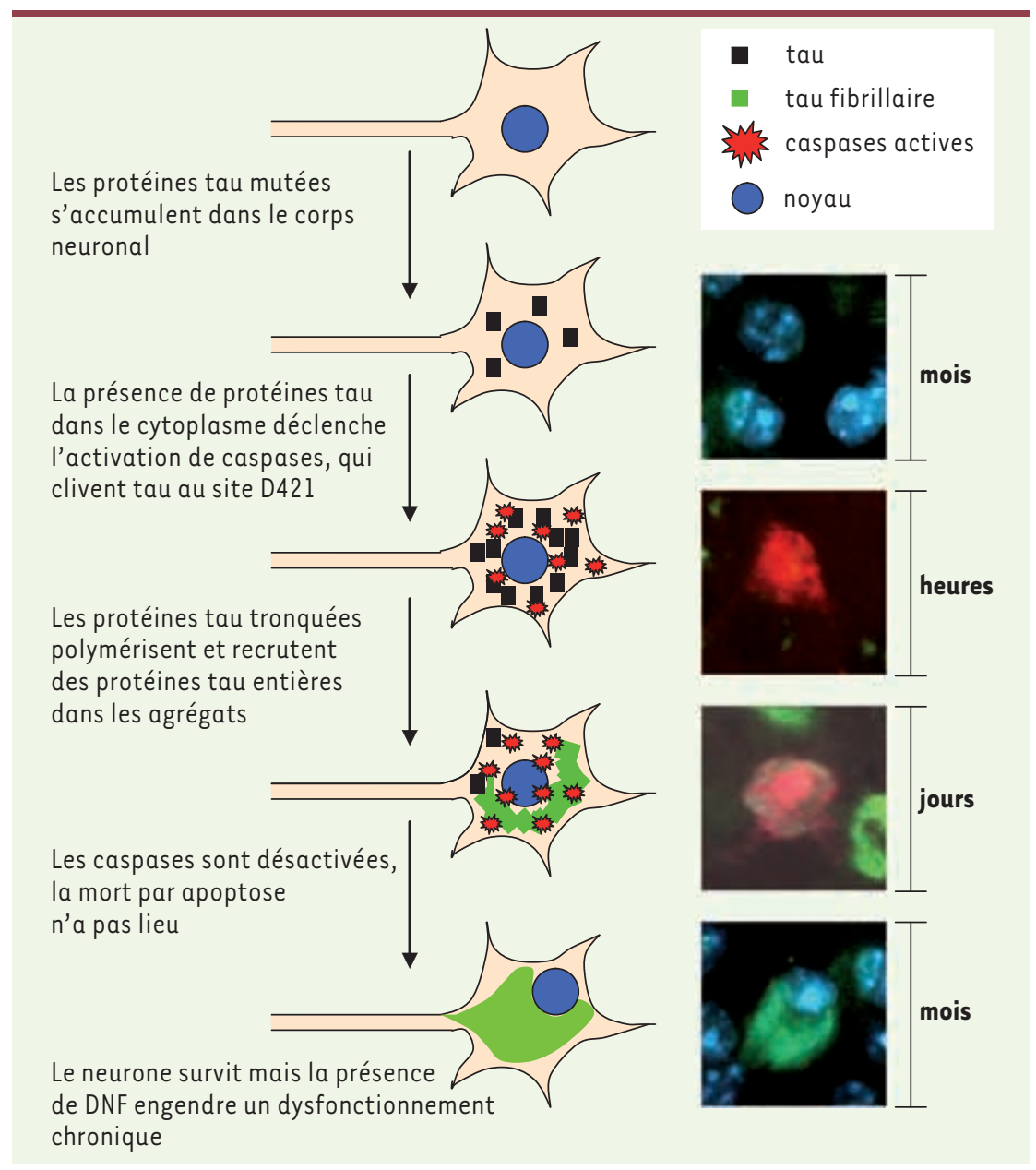

de cerveaux post-mortem, la visualisation directe de la pathologie neurofibrillaire par imagerie longitudinale a permis d'apporter des éléments importants sur la séquence des événements dans un même neurone. Nos travaux suggèrent que plutôt que d'être la cause d'une cascade neurodégénérative, les dépôts de tau fibrillaires sont une conséquence d'un processus de dégénérescence dont l'activation de caspases n'est qu'un signe passager. En effet, comme toutes les DNF apparaissent après l'activation de caspases, mais que la plupart des neurones contenant des DNF contiennent des caspases inactives, nous suggérons que les neurones porteurs de DNF survivent à une attaque et que les caspases sont rapidement inactivées.

Dans le modèle que nous proposons (Figure 2), l'expression de la protéine tau humaine mutée (P301L) dans le cerveau
DNF qui engendre, à long terme, des toxiques sur l'activité neuronale. $\nabla$

\section{Caspase activation precedes}

and leads to neurodegeneration

in a murine model

of Alzheimer's disease tité de proténes tau sous forme soluble diminue et les caspases sont inactivées, enrayant ainsi le processus d'apoptose.
Figure 2. Schéma synthétique de l'hypothèse de la dégénérescence neurofibrillaire.

\section{CONFLIT D'INTÉRÊTS}

Les auteurs déclarent n'avoir aucun conflit d'intérêts concernant les données publiées dans cet article.

\section{RÉFÉRENCES}

1. Duyckaerts C, Hauw JJ. Diagnosis and staging of Alzheimer disease. Neurobiol Aging 1997 ; 18 (suppl 4) : S33-42.

2. Hardy J, Selkoe DJ. The amyloid hypothesis of Alzheimer's disease: progress and problems on the road to therapeutics. Science 2002 ; 297 : 353-6.

3. Hutton M. Missense and splice site mutations in tau associated with FTDP-17: multiple pathogenic mechanisms. Neurology 2001; 56 (suppl 4) : S21-5.

4. Gomez-Isla T, Hollister R, West $H$, et al. Neuronal loss correlates with but exceeds neurofibrillary tangles in Alzheimer's disease. Ann Neurol 1997 ; 41 : 17-24.

5. Augustinack JC, Schneider A, Mandelkow EM, Hyman BT. Specific tau phosphorylation sites correlate with severity of neuronal cytopathology in Alzheimer's disease. Acta Neuropathol 2002; 103 : 26-35.

6. Rohn $\Pi$, Head $\varepsilon$, Nesse WH, et al. Activation of caspase- 8 in the Alzheimer's disease brain. Neurobiol Dis $2001 ; 8: 1006-16$.

7. Guo H, Albrecht S, Bourdeau M, et al. Active caspase6 and caspase-6-cleaved tau in neurophil threads, neuritic plaques, and neurofibrillary tangles of Alzheimer's disease. Am J Pathol 2004 ; 165 : 523-31.

8. Santacruz K, Lewis J, Spires T, et al. Tau suppression in a neurodegenerative mouse model improves memory function. Science 2005 ; 309: 476-81.

9. Bacskai BJ, Kajdasz ST, Christie RH, et al. Imaging of amyloid-beta deposits in brains of living mice permits direct observation of clearance of plaques with immunotherapy. Nat Med $2001 ; 7: 369-72$.

10. Guntern R, Bouras C, Hof PR, Vallet PG. An improved thioflavine $S$ method for staining neurofibrillary tangles and senile plaques in Alzheimer's disease. Experientia $1992 ; 48: 8-10$.

11. Spires-Jones TL, de Calignon A, Matsui T, et al. In vivo imaging reveals dissociation between caspase activation and acute neuronal death in tanglebearing neurons. J Neurosci 2008 ; 28 : 862-7.

12. De Calignon A, Spires-Jones TL, Pitstick R, et al. Tangle-bearing neurons survive despite disruption of membrane integrity in a mouse model of tauopathy. J Neuropathol Exp Neurol 2009; 68 : 757-61.

13. De Calignon A, Fox LM, Pitstick R, et al. Caspase activation precedes and leads to tangles. Nature 2010 ; 464 : 1201-4.

14. Clavaguera F, Goedert M, Tolnay M. Induction et propagation de la pathologie par la protéine tau chez un modèle murin de la maladie d'Alzheimer. Med Sci (Paris) $2010 ; 26: 121-4$. 